\title{
Jujuboside A attenuates norepinephrine-induced apoptosis of H9c2 cardiomyocytes by modulating MAPK and AKT signaling pathways
}

\author{
CHANG-RONG WAN $^{1 *}$, DAN-DAN HAN ${ }^{1 *}, J_{A A N-Q I N ~ X U^{1}}$, PENG YIN $^{1}$, \\ XIAO-LONG XU ${ }^{2}$, CHEN MEI $^{3}$, FENG-HUA LIU ${ }^{3}$ and ZHAO-FEI XIA ${ }^{1}$
}

\begin{abstract}
${ }^{1}$ CAU-BUA TCVM Teaching and Researching Team, College of Veterinary Medicine, China Agricultural University, Beijing 100193; ${ }^{2}$ Beijing Key Laboratory of Basic Research with Traditional Chinese Medicine on Infectious Disease, Beijing Hospital of Traditional Chinese Medicine, Capital Medical University, Beijing 100010; ${ }^{3}$ Beijing Key Laboratory of Dairy Cow Nutrition, College of Animal Science and Technology, Beijing University of Agriculture, Beijing 102206, P.R. China
\end{abstract}

Received November 17, 2016; Accepted June 27, 2017

DOI: $10.3892 / \mathrm{mmr} .2017 .7938$

\begin{abstract}
Cardiomyocyte apoptosis is closely associated with the pathogenesis of heart failure. Jujuboside A (JUA) is a type of saponin isolated from the seeds of Zizyphus jujuba. In traditional Chinese medicine, it is believed that JUA possesses multiple biological effects, including antianxiety, antioxidant and anti-inflammatory activities. The present study aimed to evaluate the effects of JUA on norepinephrine (NE)-induced apoptosis of $\mathrm{H} 9 \mathrm{c} 2$ cells and to investigate its underlying mechanisms. Rat H9c2 cardiomyocytes were pretreated with JUA and were then exposed to $\mathrm{NE}$ as an in vitro model of myocardial apoptosis. A cell viability assay, scanning electron microscopy, transmission electron microscopy, flow cytometry assay, acridine orange/ethidium bromide staining, reverse transcription-quantitative polymerase chain reaction and western blotting, all revealed that $\mathrm{NE}$ induced $\mathrm{H} 9 \mathrm{c} 2$ cell apoptosis. The results demonstrated that NE inhibited cell viability, and enhanced cell damage and apoptosis of H9c2 cells. Conversely, pretreatment with JUA was able to reverse NE-induced decreased cell viability and increased apoptosis. Furthermore,
\end{abstract}

Correspondence to: Professor Zhao-Fei Xia, CAU-BUA TCVM Teaching and Researching Team, College of Veterinary Medicine, China Agricultural University, 2 Yuan Ming Yuan West Road, Haidian, Beijing 100193, P.R. China

E-mail: zhaofeixiacau@126.com

Professor Feng-Hua Liu, Beijing Key Laboratory of Dairy Cow Nutrition, College of Animal Science and Technology, Beijing University of Agriculture, 7 Beinong Road, Changping, Beijing 102206, P.R. China

E-mail: liufenghua1209@126.com

*Contributed equally

Key words: jujuboside A, H9c2 cardiomyocytes, norepinephrine, apoptosis, mitogen-activated protein kinase, AKT
JUA suppressed upregulation of the B-cell lymphoma 2 (Bcl-2)-associated X protein/Bcl-2 ratio, and inhibited the increased protein expression levels of cleaved caspase- 3 and cleaved caspase-9 following NE exposure. However, the protein expression levels of cleaved caspase-12 and cleaved caspase- 8 were not significantly altered following exposure to NE or JUA pretreatment. In addition, in JUA-pretreated cells, the protein expression levels of phosphorylated (p)-p38 and p-c-Jun N-terminal kinase were downregulated compared with in NE-treated cells. Furthermore, JUA regulated the activation of extracellular signal-regulated kinase (ERK) in NE-treated cells and significantly increased the expression levels of p-AKT. Taken together, these data suggested that JUA may protect against NE-induced apoptosis of cardiomyocytes via modulation of the mitogen-activated protein kinase and AKT signaling pathways. Therefore, JUA may be considered a potential therapeutic strategy for the treatment of heart disease.

\section{Introduction}

Enhanced activity of the sympathetic nervous system in the myocardium is an important feature of heart failure (1). Notably, a significant loss of cardiomyocytes by apoptosis is a major pathogenic feature of various cardiovascular diseases, including heart failure, myocardial ischemia and infarction (2). Norepinephrine (NE), which is the primary transmitter in the sympathetic nervous system, has been demonstrated to induce apoptosis of adult and neonatal rat cardiomyocytes (3). Enhancement of cardiac sympathetic nerve activity in myocardial ischemia elicits the release of NE from nerve endings (4). NE-induced apoptosis of cardiomyocytes is closely associated with heart failure $(5,6)$. Our previous study indicated that transport stress resulted in an increase in NE and mitochondrial apoptosis (7). Therefore, in the present study, NE was used to stimulate $\mathrm{H} 9 \mathrm{c} 2$ cells to explore the underlying mechanism of apoptosis.

Spina date seed (SZS), which is the mature seed of Ziziphus jujuba Mill. var. spinosa (Bunge) Hu ex H.F. Chow, is traditionally used as a folk medicine, due to its anti-anxiety 
activity (8). Jujuboside A (JUA) is a classic natural product extracted from SZS, which has been demonstrated to be the most effective pharmacological active component of SZS (9). Previous studies have reported that JUA exerts antioxidant, anti-anxiety, anti-inflammatory and hypnotic-sedative activities, and is able to reduce cell apoptosis $(10,11)$. Our previous study indicated that JUA could markedly reduce the damage caused by isoproterenol via activating the phosphoinositide 3-kinase/AKT/mammalian target of rapamycin pathway (12). However, to the best of our knowledge, no previous studies have focused on the protective effects of JUA on NE-induced cardiomyocyte apoptosis.

The present study aimed to examine the protective effects of JUA on $\mathrm{H} 9 \mathrm{c} 2$ cardiomyocytes against NE-induced apoptosis in vitro, in order to elucidate the underlying mechanism of JUA. The present study investigated JUA-mediated protection against $\mathrm{NE}$-induced apoptosis, and the involvement of the mitogen-activated protein kinase (MAPK) and AKT signaling pathways.

\section{Materials and methods}

Reagents and antibodies. JUA ( $>98 \%$ purity, as determined by high-performance liquid chromatography) was purchased from the National Institutes for Food and Drug Control (Beijing, China). NE and MTT were obtained from Sigma-Aldrich (Merck KGaA, Darmstadt, Germany). High-glucose Dulbecco's modified Eagle's (DMEM), fetal bovine serum (FBS) and antibiotic-antimycotic were purchased from Gibco (Thermo Fisher Scientific, Inc., Waltham, MA, USA). Bicinchoninic acid (BCA) protein assay kit was purchased from Pierce (Thermo Fisher Scientific, Inc.). Antibodies against GAPDH (cat. no. 5174S), c-Jun N-terminal kinase (JNK; cat. no. 9258), phosphorylated (p)-JNK (cat. no. 4668), p38 (cat. no. 9212), p-p38 (cat. no. 9215), extracellular signal-regulated kinase (ERK; cat. no. 4695), p-ERK (cat. no. 4370), AKT (cat. no. 4685), p-AKT (cat. no. 4060), cleaved caspase-3 (cat. no. 9661), cleaved caspase- 8 (cat. no. 8592), cleaved caspase- 9 (cat. no. 7237) and cleaved caspase-12 (cat. no. 2202) were purchased from Cell Signaling Technology, Inc. (Danvers, MA, USA). Goat anti-rabbit secondary antibody was obtained from LI-COR Biotechnology (Lincoln, NE, USA; cat. no. 925-32211).

Cell culture and treatment. H9c2 cells were purchased from the Cell Resource Center of Chinese Academy of Medical Sciences (Peking Union Medical College, Beijing, China). Cells were cultured in DMEM supplemented with $10 \%$ FBS and antibiotics $(100 \mathrm{U} / \mathrm{ml}$ penicillin and $100 \mathrm{U} / \mathrm{ml}$ streptomycin) at $37^{\circ} \mathrm{C}$ in a humidified atmosphere containing $5 \%$ $\mathrm{CO}_{2}$. The cells were subcultured with $0.05 \%$ trypsin (Gibco; Thermo Fisher Scientific, Inc.). JUA and NE were diluted in DMEM. H9c2 cells were washed with phosphate-buffered saline (PBS) twice and serum-starved for $2 \mathrm{~h}$ prior to incubation with JUA or NE.

Cell viability assay. Cell viability was determined using the MTT reduction assay as previously described (12). Briefly, H9c2 cells were preincubated with DMEM containing $10 \%$ FBS overnight in 96-well plates at a density of $5 \times 10^{4}$ cells/well.
After reaching $85 \%$ confluence, the cells were washed twice with PBS and incubated with medium containing various concentrations of JUA $(0,2,5,10,20,50$ and $100 \mu \mathrm{M})$ for $3,24,48$ and $72 \mathrm{~h}$ prior to treatment with or without $\mathrm{NE}$ $(5 \mu \mathrm{M})$ for 6,12 and $18 \mathrm{~h}$ at $37^{\circ} \mathrm{C}$ in a humidified atmosphere containing $5 \% \mathrm{CO}_{2}$. The medium was removed, and $100 \mu \mathrm{l}$ DMEM containing $10 \% \mathrm{MTT}(0.5 \mathrm{mg} / \mathrm{ml})$ was added to each well for $4 \mathrm{~h}$. The formazan crystals that formed in intact cells were dissolved in $200 \mu \mathrm{l}$ dimethyl sulfoxide. Absorbance was recorded at a wavelength of $490 \mathrm{~nm}$, and at a reference wavelength of $630 \mathrm{~nm}$, using a microplate reader (Bio-Rad Laboratories, Inc., Hercules, CA, USA).

Morphological observations. Cells were treated with or without $\mathrm{NE}(0$ and $5 \mu \mathrm{M})$ for $12 \mathrm{~h}$ at $37^{\circ} \mathrm{C}$ in a humidified atmosphere containing $5 \% \mathrm{CO}_{2}$. Following treatment, cell morphology was observed under a phase-contrast inverted biological microscope (IX71/IX2; Olympus Corporation, Tokyo, Japan), and by scanning electron microscopy (SEM; S-3400 N, Hitachi, Ltd., Tokyo, Japan) and transmission electron microscopy (TEM; 1230, JEOL, Ltd., Tokyo, Japan). For ultrastructural studies, $\mathrm{H} 9 \mathrm{c} 2$ cells were harvested and fixed with $3.0 \%$ glutaraldehyde and $1.5 \%$ paraldehyde, washed three times in PBS, and post-fixed in cold $1 \%$ osmium tetroxide. Following dehydration with a graded series of alcohol, all samples were freeze-dried, coated with a layer of gold using a sputter-coater and embedded in epoxy resin (EPON812; Beijing Solarbio Science \& Technology Co., Ltd., Beijing, China). Subsequently, the cells were examined by SEM using an Hitachi S-3400 N operated at $15 \mathrm{kV}$. Following dehydration with a graded series of alcohol, samples were embedded in 50/50 LR White Embedding Resin (Electron Microscopy Sciences, Hatfield, PA, USA) and treated with pure ethanol solution for $15 \mathrm{~min}$ at $37^{\circ} \mathrm{C}$, then with a pure resin solution overnight at $4^{\circ} \mathrm{C}$ followed by incubation at $60^{\circ} \mathrm{C}$ for $1 \mathrm{~h}$ for polymerization. Thin slices $(80 \mathrm{~nm})$ were produced using an Ultracut Microtome (Leica EM UC7 Ultramicrotome; Leica Microsystems GmbH, Wetzlar, Germany). Ultra-thin sections were stained with saturated uranyl acetate in 50\% ethanol and lead citrate, and $\mathrm{H} 9 \mathrm{c} 2$ cardiomyocyte ultrastructure was examined by TEM.

Annexin V-fluorescein isothiocyanate (FITC)/propidium iodide (PI) apoptotic analysis. Cellular apoptosis was determined using the Annexin V-FITC/PI cell apoptosis detection kit (Thermo Fisher Scientific, Inc.). Following treatment, the cells were harvested by trypsinization, washed twice with cold PBS and were centrifuged at 1,200 x g for $10 \mathrm{~min}$ at $4^{\circ} \mathrm{C}$. Cells were resuspended in $100 \mu \mathrm{l} 1 \mathrm{X}$ binding buffer and were transferred to a sterile flow cytometry glass tube. Subsequently, $5 \mu 1$ Annexin V-FITC and $5 \mu \mathrm{l}$ propidium iodide were added and incubated at room temperature $\left(25^{\circ} \mathrm{C}\right)$ in the dark for $15 \mathrm{~min}$. Finally, detection was performed by flow cytometry (Beckman Coulter, Inc., Brea, CA, USA) according to the manufacturer's protocol, and data was analyzed using FCS Express software (version 3.0; De Novo Software, Glendale, CA, USA).

Acridine orange (AO)/ethidium bromide (EB) staining. The apoptotic morphology of $\mathrm{H} 9 \mathrm{c} 2$ cells was detected by staining the cells with a combination of the fluorescent DNA-binding 
dyes AO and EB (100 $\mu \mathrm{g} / \mathrm{ml}$ each in PBS; Sigma-Aldrich; Merck KGaA) for $15 \mathrm{~min}$ at room temperature. Subsequently, the cells were observed under a fluorescence microscope (IX71/IX2; Olympus Corporation).

$R N A$ extraction and reverse transcription-quantitative polymerase chain reaction (RT-qPCR) analysis. Total RNA was isolated from $\mathrm{H} 9 \mathrm{c} 2$ cells using the phenol and guanidine isothiocyanate-based TRIzol reagent (Invitrogen; Thermo Fisher Scientific, Inc.). RNA concentration and purity were determined using a spectrophotometer (SmartSpec Plus; Bio-Rad Laboratories, Inc.) based on the ratios of optical density $(\mathrm{OD})_{260} / \mathrm{OD}_{280}$ and $\mathrm{OD}_{260} / \mathrm{OD}_{230}$. Total mRNA was reverse transcribed using the iScript cDNA synthesis kit (Promega Corporation, Madison, WI, USA) according to the manufacturer's protocol. The expression levels of Bax and Bcl-2 were determined by qPCR analysis using the DNA Engine Mx3000P ${ }^{\circledR}$ fluorescence detection system (Stratagene; Agilent Technologies, Inc., Santa Clara, CA, USA). The designed paired primers were as follows: $\beta$-actin, forward 5'-CCTGCGGCATTCACGAAACTAC-3' and reverse 5'-ACTCCTGCTTGCTGATCCACATC-3'; B-cell lymphoma 2(Bcl-2)-associated X protein (Bax), forward 5'-CAGGAC GCATCCACCAAGAA-3', reverse 5'-GGGTCCCGAAGT AGGAAAGG-3'; and Bcl-2, forward 5'-CTGGGAGAA CAGGGTATG-3' and reverse 5'-CGTAGAAGAGGAGGG TC-3'. RT-qPCR analysis was performed using the SYBR PrimeScript ${ }^{\mathrm{TM}}$ RT-PCR kit (Takara Biotechnology Co., Ltd., Dalian, China). The thermocycling conditions were as follows: $94^{\circ} \mathrm{C}$ for $5 \mathrm{~min}$, followed by 40 cycles of $94^{\circ} \mathrm{C}$ for $10 \mathrm{sec}, 60^{\circ} \mathrm{C}$ for $20 \mathrm{sec}$ and $72^{\circ} \mathrm{C}$ for $60 \mathrm{sec}$. RNA expression was quantified using the $2^{-\Delta \Delta C q}$ relative quantification method (13).

Western blot analysis. Protein was extracted from the cells using a total protein extraction kit (Biochain Institute, Inc., Newark, CA, USA), and was quantified using a BCA protein assay kit according to the manufacturer's protocol. Proteins $(20 \mu \mathrm{g})$ were separated by $12 \%$ SDS-PAGE and were transferred to nitrocellulose membranes (Pierce; Thermo Fisher Scientific, Inc.). Subsequently, membranes were blocked with SuperBlock T20 (TBS) blocking buffer (cat. no. 37536, Pierce, USA) for $2.5 \mathrm{~h}$ at room temperature, and were then incubated overnight at $4^{\circ} \mathrm{C}$ with the specific primary antibodies to GAPDH $(1: 1,000)$, JNK $(1: 1,000)$, p-JNK $(1: 1,000)$, p38 (1:1,000), p-p38 (1:1,000), ERK $(1: 1,000)$, p-ERK $(1: 2,000)$, AKT $(1: 1,000)$, p-AKT $(1: 2,000)$, cleaved caspase-3 $(1: 1,000)$, cleaved caspase-8 $(1: 1,000)$, cleaved caspase- 9 $(1: 1,000)$ and cleaved caspase-12 $(1: 1,000)$. The membranes were then incubated with the secondary antibody $(1: 15,000)$ for $1 \mathrm{~h}$ at room temperature. Subsequently, the blots were visualized and analyzed using the Odyssey Infrared Imaging system (LI-COR Biotechnology). Blots were normalized to GAPDH and were semi-quantified using ImageJ version 2.1.4.7 software (National Institutes of Health, Bethesda, MD, USA).

Statistical analysis. Data are presented as the mean \pm standard error of the mean of at least three independent experiments. The results were analyzed by one-way analysis of variance followed by Duncan's test for multiple comparisons using SPSS
19.0 (IBM Corp., Armonk, NY, USA). P $<0.05$ was considered to indicate a statistically significant difference.

\section{Results}

NE inhibits cell viability, enhances apoptosis and induces morphological alterations in $\mathrm{H} 9 \mathrm{c} 2$ cells. H9c2 cells were treated with various concentrations of $\mathrm{NE}$ for $0,6,12$, and $18 \mathrm{~h}$. The results of an MTT assay demonstrated that the number of viable cells decreased in response to the increased concentration and duration of NE treatment. (Fig. 1A). Following stimulation with 20 or $50 \mu \mathrm{M} \mathrm{NE}$ for $6 \mathrm{~h}$, cell viability was significantly inhibited $(\mathrm{P}<0.05$; Fig. $1 \mathrm{~A})$. A more marked decrease $(\mathrm{P}<0.01$; Fig. $1 \mathrm{~A})$ in cell viability occurred following treatment with NE for 12 or $18 \mathrm{~h}$. Furthermore, flow cytometric analysis demonstrated that the apoptotic rate of $\mathrm{H} 9 \mathrm{c} 2$ cells was increased following treatment with $5 \mu \mathrm{M}$ $\mathrm{NE}$ for $12 \mathrm{~h}$ (Fig. 1B). As observed under SEM, NE-treated cells exhibited an abnormal cellular microstructure, which was characterized by irregular-arranged cell shape, sparser cell surface and increased nuclear gap (Fig. 1C). Electron microscopy analysis of $\mathrm{H} 9 \mathrm{c} 2$ cells revealed marked alterations in the structure of cardiomyocytes and the cellular architecture. Ultrastructural images of H9c2 cells using TEM showed obvious nuclear chromatin margination, aggregation, condensation, and mitochondrial vacuolization in NE-treated cells (Fig. 1D). According to these results, treatment with $5 \mu \mathrm{M} \mathrm{NE}$ for $12 \mathrm{~h}$ was selected for the generation of an in vivo model of H9c2 cell damage.

Cytotoxic effects of JUA on $\mathrm{H} 9 \mathrm{c} 2$ cells. $\mathrm{H} 9 \mathrm{c} 2$ cells were treated with various concentrations of JUA $(0,2,5,10,20,50$ and $100 \mu \mathrm{M}$ ) for 24,48 and $72 \mathrm{~h}$. Cell viability was determined using an MTT assay. The results demonstrated that treatment with between 0 and $100 \mu \mathrm{M}$ JUA had no cytotoxic effect on H9c2 cells (Fig. 2).

JUA improves cell viability and reduces $H 9 c 2$ apoptosis following NE exposure. The results of an MTT assay demonstrated that JUA significantly enhanced the survival rate of the cells following exposure to NE (Fig. 3A). In addition, flow cytometry indicated that JUA reduced apoptosis in a dose-dependent manner at $12 \mathrm{~h}$ following NE exposure (Fig. 3B and C). Pretreatment with JUA was also revealed to alleviate the apoptotic response in $\mathrm{H} 9 \mathrm{c} 2$ cells following treatment with NE, as determined by AO and EB staining (Fig. 3D).

JUA initially upregulates the $\mathrm{Bax} / \mathrm{Bcl}-2$ ratio and then downregulates the ratio following treatment of $\mathrm{H} 9 \mathrm{c} 2$ cells with NE. The results of an RT-qPCR demonstrated that the mRNA expression levels of Bax were upregulated following exposure to NE for 3 and $6 \mathrm{~h}$; this effect was markedly inhibited by JUA (Fig. 4A). In addition, pretreatment with JUA was able to downregulate the mRNA expression levels of Bcl-2 at 3 and $6 \mathrm{~h}$ following NE exposure, and upregulate the mRNA expression levels of Bcl-2 at 9 and $12 \mathrm{~h}$ following NE exposure (Fig. 4B). Correspondingly, the ratio of Bax/Bcl-2 in JUA-pretreated cells was increased following exposure to NE for 3 and $6 \mathrm{~h}$ (with all JUA 

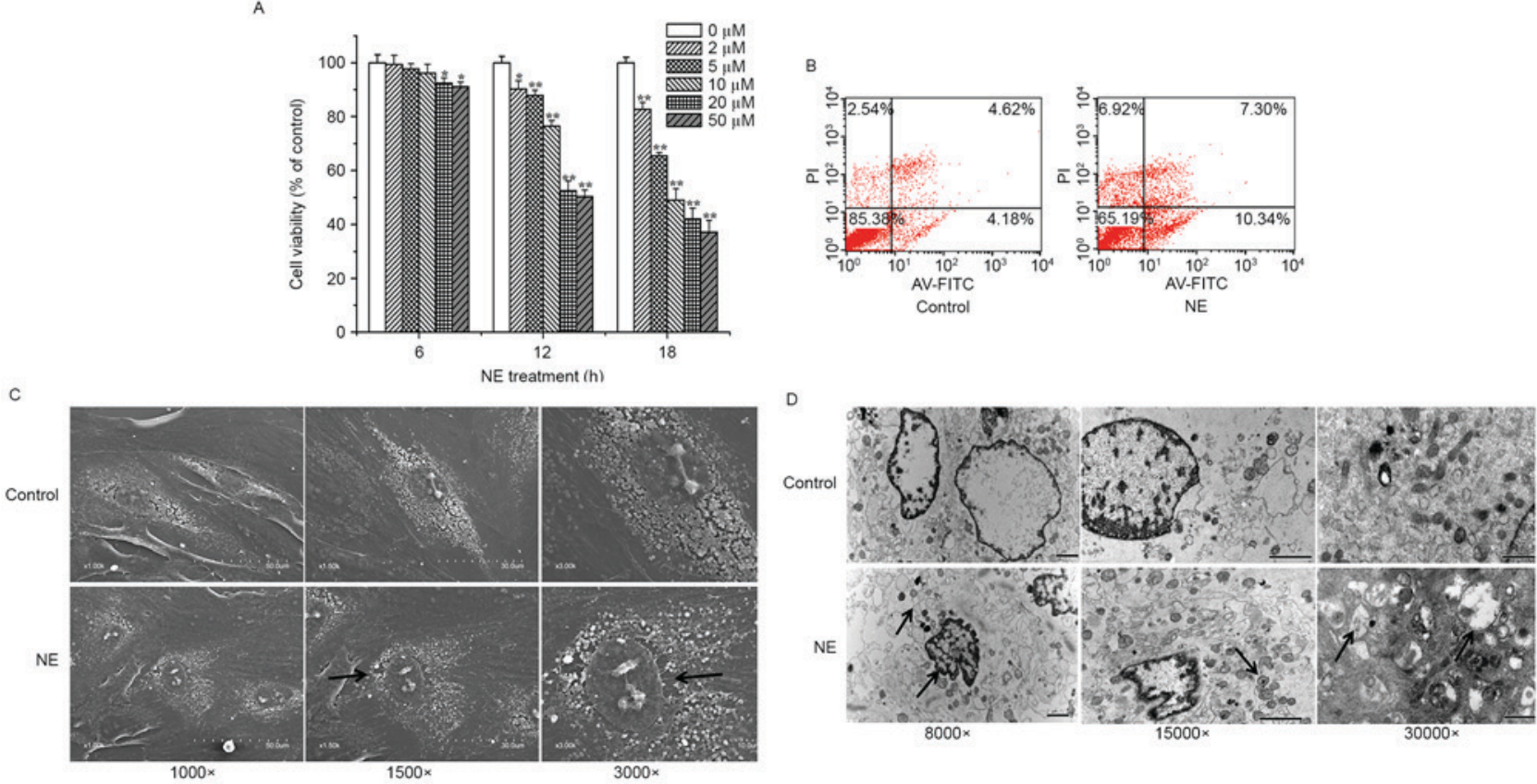

Figure 1. Effects of NE on cell viability, ultrastructural alterations and apoptosis of H9c2 cells. (A) Cell viability was measured using an MTT assay following treatment with various concentrations $(0,2,5,10,20$ and $50 \mu \mathrm{M})$ of NE for 6,12 and $18 \mathrm{~h}$. (B-D) Cells were treated with or without NE $(5 \mu \mathrm{M})$ for $12 \mathrm{~h}$. (B) Apoptosis was measured by flow cytometry, following AV-FITC (FL 1 channels) and PI (FL 2 channels) double staining. Morphological characteristics of H9c2 cells were observed using a (C) scanning electron microscope and a (D) transmission electron microscope. Black arrows indicate the following: (C) sparser cell surface (x1,500 image) and increased nuclear gap (x3,000 image); (D) nuclear chromatin margination and swelling of endoplasmic reticulum (x8,000 image), mitochondrial (x15,000 image) and mitochondrial vacuolization (x30,000 image). Data are presented as the mean \pm standard error of the mean $(\mathrm{n}=6) .{ }^{*} \mathrm{P}<0.05,{ }^{* *} \mathrm{P}<0.01$ compared with the control group. AV-FITC, Annexin V-fluorescein isothiocyanate; NE, norepinephrine; PI, propidium iodide.

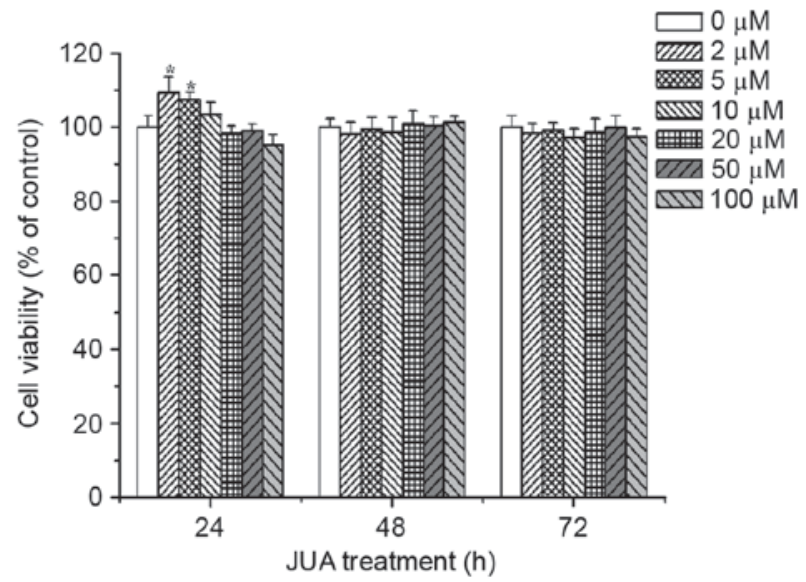

Figure 2. Cytotoxic effects of JUA on H9c2 cells. The effects of JUA on the viability of $\mathrm{H} 9 \mathrm{c} 2$ cells were measured using an MTT assay. Cells were treated with various concentrations of JUA $(0,2,5,10,20,50$ and $100 \mu \mathrm{M})$ for 24,48 and $72 \mathrm{~h}$. Data are presented as the mean \pm standard error of the mean $(n=6) .{ }^{*} \mathrm{P}<0.05$, compared with the control group. JUA, jujuboside A.

pretreatments), and was decreased after exposure to NE for $9 \mathrm{~h}$ (with all JUA pretreatments) and $12 \mathrm{~h}$ (5 and $20 \mu \mathrm{M} \mathrm{JUA}$ pretreatments only). However, the Bax/Bcl-2 ratio increased following JUA $(10 \mu \mathrm{M})$ pretreatment and NE incubation for $12 \mathrm{~h}$ (Fig. 4C). With the prolonged incubation time with $\mathrm{NE}$, the mRNA expression levels of the anti-apoptotic gene Bcl-2 were downregulated, whereas the expression levels of the proapoptotic gene Bax were upregulated, thus suggesting that JUA may exert its anti-apoptotic effect by regulating the ratio of $\mathrm{Bax} / \mathrm{Bcl}-2$.
JUA inhibits NE-induced cleaved caspase-3 and cleaved caspase-9 protein expression in $\mathrm{H} 9 \mathrm{c} 2$ cells. Western blotting revealed that the protein expression levels of cleaved caspase-3 and cleaved caspase- 9 were significantly increased in cells treated with NE for 6 and $12 \mathrm{~h}$, whereas pretreatment with JUA significantly decreased the protein expression levels of cleaved caspase- 3 and cleaved caspase- 9 in the cells (Fig. 5A-C). However, the protein expression levels of cleaved caspase-12 and cleaved caspase- 8 were not significantly altered following exposure to NE or JUA pretreatment (Fig. 5A, D and E), thus indicating that JUA may alleviate NE-induced apoptosis via the mitochondrial-dependent pathway in H9c2 cardiomyocytes.

JUA reduces NE-induced apoptosis via influencing MAPK and AKT signals in $\mathrm{H} 9 \mathrm{c} 2$ cells. To further elucidate the signaling pathways by which JUA exerts its anti-apoptotic effects, the activation of MAPK and AKT were examined. Western blotting results demonstrated that the expression levels of p-JNK and p-p38 were significantly increased following exposure to NE for 6 and $12 \mathrm{~h}$. Conversely, pretreatment with JUA was able to reduce the expression levels of p-JNK and p-p38 at 6 or $12 \mathrm{~h}$ following NE exposure (Fig. 6A-C). In addition, the expression levels of p-ERK were significantly increased following treatment with NE. Pretreatment with JUA significantly reduced the expression levels of p-ERK at $6 \mathrm{~h}$ following NE exposure. However, in cells pretreated with $20 \mu \mathrm{M}$ JUA, the expression levels of p-ERK were significantly increased at $12 \mathrm{~h}$ following NE exposure (Fig. 6A and D). Furthermore, in the groups receiving 10 and $20 \mu \mathrm{M}$ JUA, the expression levels of p-AKT were significantly increased at 6 or $12 \mathrm{~h}$ following 


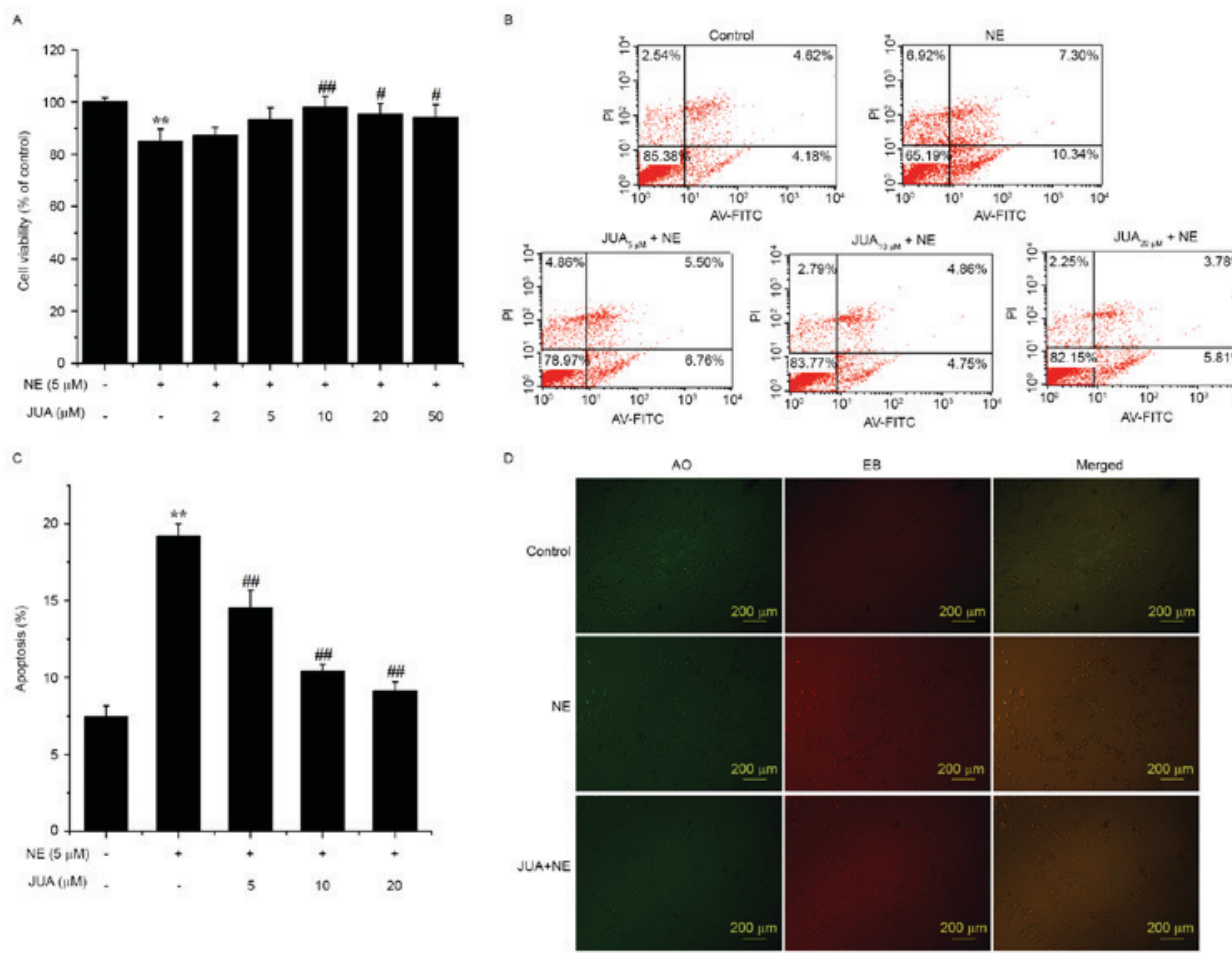

Figure 3. Effects of JUA on NE-induced cytotoxicity and apoptosis of H9c2 cells. Cells were treated with or without JUA at the indicated concentrations for $3 \mathrm{~h}$ and then incubated with NE $(5 \mu \mathrm{M})$ for a further $12 \mathrm{~h}$. (A) Cell viability was determined using an MTT assay. (B-D) Apoptosis was detected by flow cytometry and AO/EB staining. Data are presented as the mean \pm standard error of the mean $(\mathrm{n}=6)$, ${ }^{* *} \mathrm{P}<0.01$, compared with the control group; ${ }^{\#} \mathrm{P}<0.05$, ${ }^{\# \#} \mathrm{P}<0.01$, compared with the NE-treated group. AO, acridine orange; AV-FITC, Annexin V-fluorescein isothiocyanate; EB, ethidium bromide; JUA, jujuboside A; NE, norepinephrine; PI, propidium iodide.
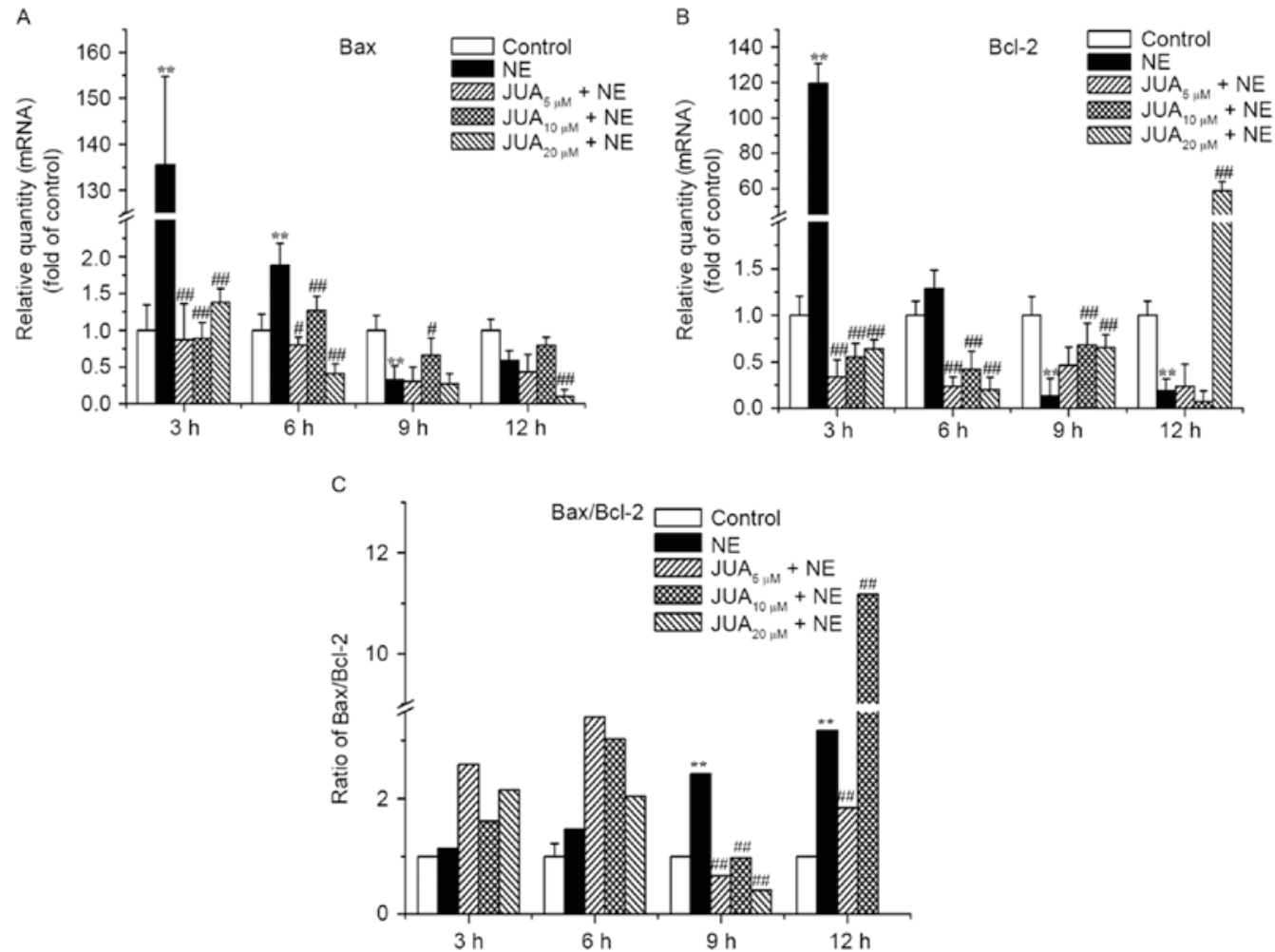

Figure 4. Effects of JUA on NE-induced Bax and Bcl-2 mRNA expression in H9c2 cells. Cells were pretreated with or without JUA at the indicated concentrations for $3 \mathrm{~h}$ and were then incubated with NE $(5 \mu \mathrm{M})$ for 3, 6, 9 and $12 \mathrm{~h}$. Total RNA was isolated at the indicated time points and analyzed by RT-qPCR RT-qPCR analysis was used to detect the mRNA expression levels of (A) Bax and (B) Bcl-2. (C) Ratio of Bax/Bcl-2 was calculated according to the difference in mRNA expression. Data are presented as the mean \pm standard error of the mean $(n=3)$. ${ }^{* *} \mathrm{P}<0.01$, compared with the control group; ${ }^{\#} \mathrm{P}<0.05,{ }^{\# /} \mathrm{P}<0.01$, compared with the NE-treated group. Bax, B-cell lymphoma 2-associated X protein; Bcl-2, B-cell lymphoma 2; JUA, jujuboside A; NE, norepinephrine; RT-qPCR, reverse transcription-quantitative polymerase chain reaction. 
A

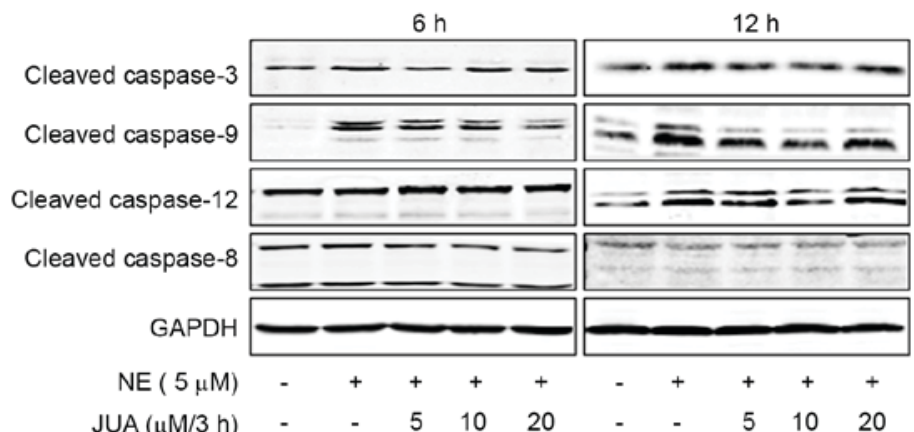

B

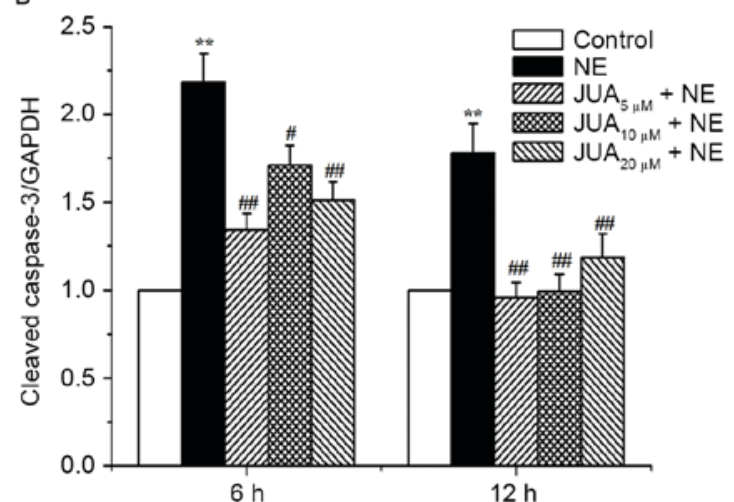

D

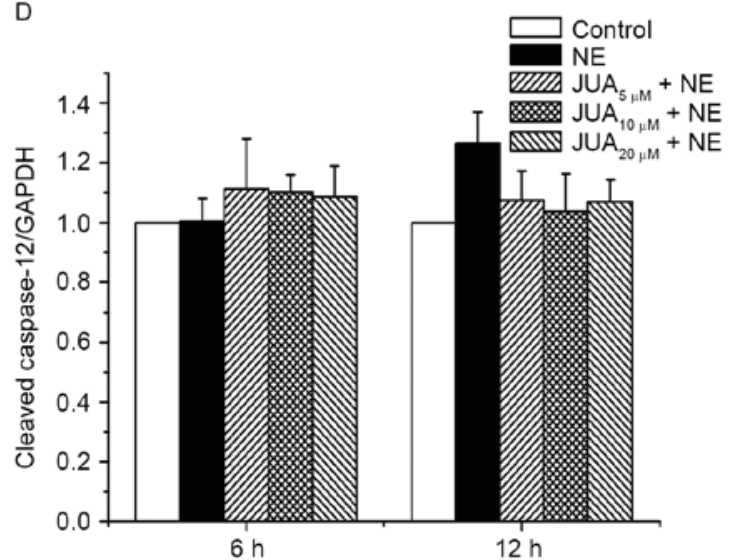

C
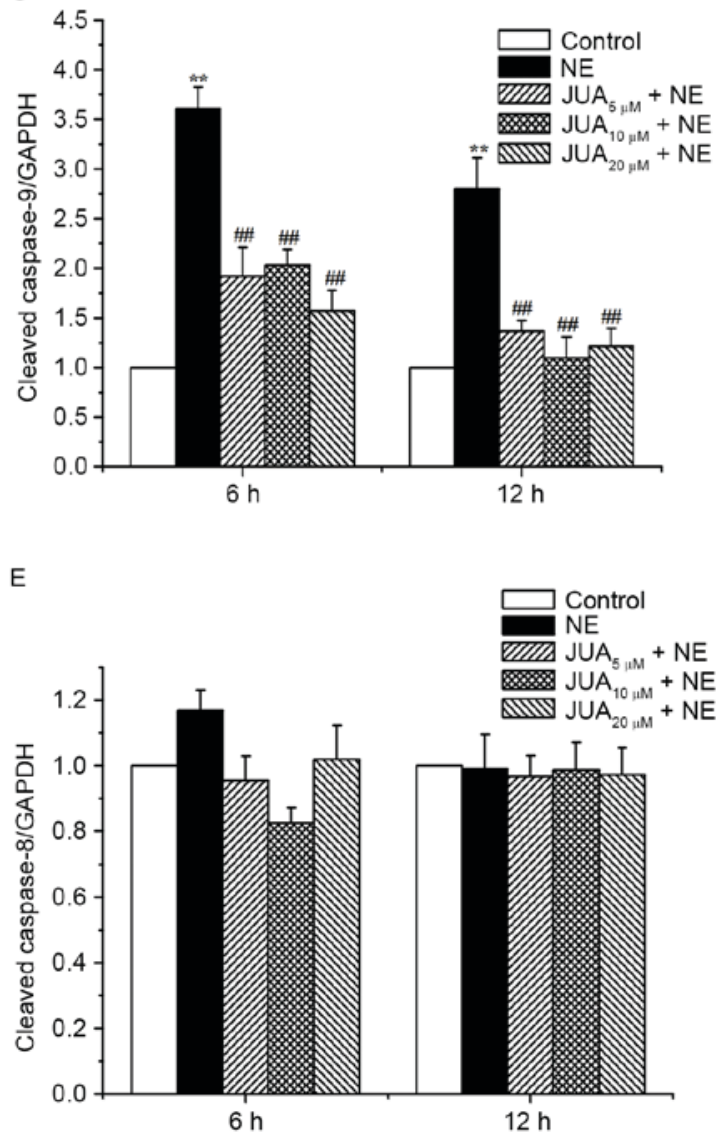

Figure 5. Effects of JUA on NE-induced cleaved caspase-3, cleaved caspase-9, cleaved caspase-12 and cleaved caspase-8 protein expression in H9c2 cells. Cells were pretreated with or without JUA at the indicated concentrations for $3 \mathrm{~h}$ and were then incubated with NE $(5 \mu \mathrm{M})$ for 6 and $12 \mathrm{~h}$. Total proteins were extracted at the indicated time points and analyzed by western blotting. (A) Proteins were subjected to western blotting with antibodies against cleaved caspase-3, cleaved caspase-9, cleaved caspase-12 and cleaved caspase-8 (B-E). Relative density of the bands was normalized to GAPDH and semi-quantification of the blots, conducted by densitometry, are shown in (B-E). Data are presented as the mean \pm standard error of the mean $(\mathrm{n}=3),{ }^{* * *} \mathrm{P}<0.01$, compared with the control group; ${ }^{\#} \mathrm{P}<0.05,{ }^{\#} \mathrm{P}<0.01$, compared with the NE-treated group. JUA, jujuboside A; NE, norepinephrine.

NE exposure (Fig. 6A and E). The total expression levels of JNK, p38, ERK and AKT were not significantly altered following exposure to NE or JUA pretreatment.

\section{Discussion}

The majority of in vitro and in vivo studies have demonstrated that NE-induced cardiotoxicity is a major mediator of the biochemical alterations leading to cardiomyocyte apoptosis and necrosis (14-17). Our previous study revealed that increased plasma NE and epinephrine levels in stress-induced rats promoted myocardial damage and cardiac dysfunction (6). Therefore, the present study exposed H9c2 cells to
$\mathrm{NE}$, and subsequently assessed cell viability, apoptosis and the morphological alterations of $\mathrm{H} 9 \mathrm{c} 2$ cell microstructure. The results demonstrated that the survival rate of $\mathrm{H} 9 \mathrm{c} 2$ cells was decreased in a time- and dose-dependent manner following exposure to NE. Furthermore, marked NE-induced cell damage and apoptosis were detected in H9c2 cells. These findings verified that NE induced cell damage and apoptosis of $\mathrm{H} 9 \mathrm{c} 2$ cells.

JUA is a natural product isolated from the seeds of Zizyphus jujuba, which possesses numerous biological effects (8). Previous studies have reported that JUA exerts anti-injury effects, and neuroprotective and cardioprotective activity via antioxidative and anti-inflammatory effects in 

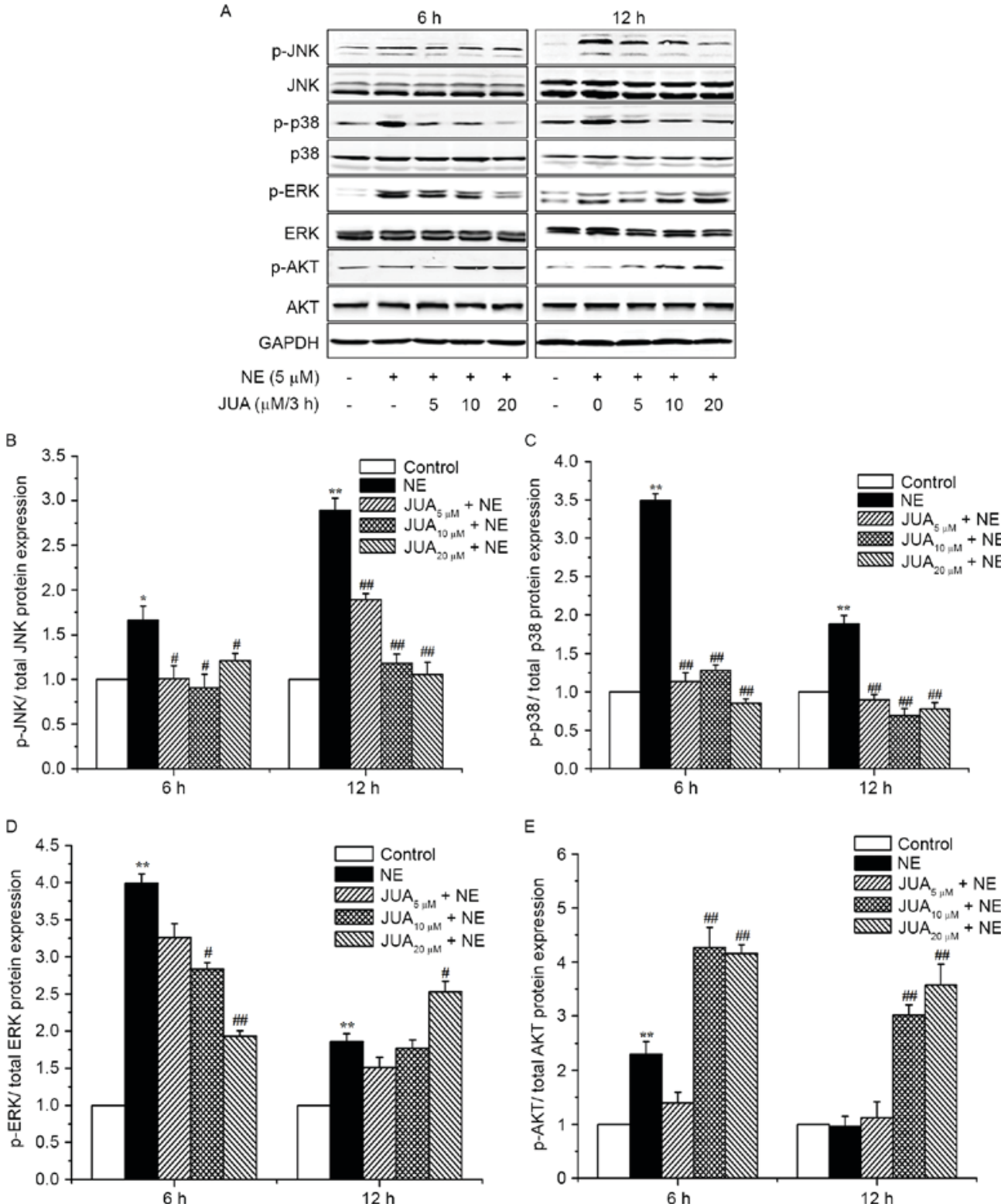

Figure 6. Effects of JUA on NE-induced JNK, p38-MAPK, ERK and AKT protein expression in H9c2 cells. Cells were pretreated with or without JUA at the indicated concentrations for $3 \mathrm{~h}$ and were then incubated with NE $(5 \mu \mathrm{M})$ for a further 6 and $12 \mathrm{~h}$. (A) Total proteins were prepared at the indicated time points and were then subjected to western blotting with antibodies against the total and phosphorylated forms of JNK, p38, ERK and AKT. (B-E) Semi-quantitative results of western blot analysis used to determine p-JNK, p-p38, p-ERK and p-AKT expression. Data are presented as the mean \pm standard error of the mean $(\mathrm{n}=3),{ }^{*} \mathrm{P}<0.05,{ }^{* *} \mathrm{P}<0.01$, compared with the control group; ${ }^{\#} \mathrm{P}<0.05,{ }^{\# \#} \mathrm{P}<0.01$, compared with the NE-treated group. ERK, extracellular signal-regulated kinase; JNK, c-Jun N-terminal kinase; JUA, jujuboside A; NE, norepinephrine; p-, phosphorylated.

animal models of dementia $(10,12,18,19)$. In the present study, the results of an MTT assay indicated that JUA $(0-100 \mu \mathrm{M})$ did not exhibit a cytotoxic effect on $\mathrm{H} 9 \mathrm{c} 2$ cells. Furthermore, JUA effectively reversed the decreased cell viability caused by NE and reduced $\mathrm{NE}$-induced $\mathrm{H} 9 \mathrm{c} 2$ cell apoptosis.

It has previously been reported that NE may induce cell apoptosis through activating the $\mathrm{Bax} / \mathrm{Bcl}$-extra large/caspase-3 pathway, the $\mathrm{Bcl}-2 /$ caspase- 2 pathway, the $\beta$-adrenergic pathway and the p38 MAPK pathway $(3,20)$. The present study confirmed that NE activated the p38 MAPK pathway, which was consistent with previous research (21). In addition, the Bax and Bcl-2 mRNA expression levels were detected in the present study. Bax is a proapoptotic member of the $\mathrm{Bcl}-2$ protein family that resides in the outer mitochondrial membrane (22). A change in the Bax/Bcl-2 ratio may contribute to an increase in caspase-3 activity via the release of cytochrome $c$ from the mitochondria (23). The release of cytochrome $c$ initiates the deoxyadenosine triphosphate-dependent oligomerization of apoptotic protease activating factor 1 and caspase- 9 to form the apoptosome, which can further activate effector caspase-3, resulting in cell apoptosis (24). The present study revealed that the Bax/Bcl-2 ratio was not significantly altered at 3 and $6 \mathrm{~h}$ following NE exposure, however, it was upregulated at 9 and $12 \mathrm{~h}$ following NE exposure. This regulation was attenuated by JUA, with exception to cells pretreated with $10 \mu \mathrm{M}$ JUA and NE for $12 \mathrm{~h}$, which produced a significant increase in the $\mathrm{Bax} / \mathrm{Bcl}-2$ ratio when compared with the NE group. The increase in the $\mathrm{Bax} / \mathrm{Bcl}-2$ ratio observed in cells treated with $\mathrm{NE}$ for 9 and $12 \mathrm{~h}$ indicated that $\mathrm{H} 9 \mathrm{c} 2$ cell apoptosis may occur over a long period time. In addition, JUA reversed NE-induced 
increases in the protein expression levels of cleaved caspase-3 and cleaved caspase-9, whereas the protein expression levels of cleaved caspase- 8 and cleaved caspase-12 were not significantly altered. Bax/Bcl-2, cleaved caspase-3 and cleaved caspase- 9 have been reported to serve important roles in the mitochondrial apoptotic pathway; however, cleaved caspase- 8 and cleaved caspase-12 are key proteins in the death receptor signaling pathway and endoplasmic reticulum apoptotic pathway, respectively (25-28). The results of the present study suggested that the anti-apoptotic effects of JUA may be mediated through the mitochondrial-dependent apoptotic pathway.

MAPKs regulate various cellular responses to environmental stimuli, including cell survival, transformation and apoptosis, and serve important roles in cardiomyocyte apoptosis (29-31). ERK protects cardiomyocytes from apoptosis, whereas p38 MAPK is involved in the induction of cardiomyocyte apoptosis $(32,33)$. Furthermore, JNK signaling exerts pro- and anti-apoptotic effects; however, the proapoptotic effects appear to be most dominant in the majority of experimental models $(34,35)$. AKT exerts direct effects on apoptosis, and AKT activation is able to suppress the apoptotic effects of activated JNK and P38 MAPK signaling pathways (36). In addition, AKT has been reported to be involved in apoptosis and endoplasmic reticulum stress in the rat small intestine (37). Furthermore, JNK has been reported to negatively regulate AKT activation (38). The present study indicated that $\mathrm{NE}$ upregulated p-JNK and p-p38 expression in H9c2 cells, and that this regulation was attenuated by JUA. Treatment with NE for $6 \mathrm{~h}$ also upregulated p-AKT expression, however, JUA pretreatment further increased expression. Conversely, ERK was inhibited in JUA-pretreated cells at $6 \mathrm{~h}$ following NE exposure, whereas it was activated at $12 \mathrm{~h}$ following NE exposure, which suggested that JUA may attenuate the upregulation of ERK induced by NE during the early stages and ERK may adaptively respond to NE treatment in these short time periods. These results indicated that JUA may reduce NE-induced apoptosis by inhibiting the JNK and p38 signaling pathways, regulating the ERK pathway and activating the AKT pathway in $\mathrm{H} 9 \mathrm{c} 2$ cells.

In conclusion, the present study provided a feasible molecular explanation regarding the inhibitory effects of JUA on NE-induced apoptosis of H9c2 cells, which may offer a therapeutic option for preventing NE-induced heart failure. Further investigations are required to elucidate the precise mechanisms underlying the protective effects of JUA on cardiomyocyte apoptosis.

\section{Acknowledgements}

The present study was supported by grants from the Ministry of Agriculture, Public Service Sectors Agriculture Research Projects (grant no. 201403051-07) and the National Natural Science Foundation of China (grant nos. 31572584 and 31272478).

\section{References}

1. Ahuja P, Sdek P and MacLellan WR: Cardiac myocyte cell cycle control in development, disease, and regeneration. Physiol Rev 87: 521-544, 2007.

2. Liu B, Che W, Xue J, Zheng C, Tang K, Zhang J, Wen J and Xu Y: SIRT4 prevents hypoxia-induced apoptosis in $\mathrm{H} 9 \mathrm{c} 2$ cardiomyoblast cells. Cell Physiol Biochem 32: 655-662, 2013.
3. Lai KB, Sanderson JE and Yu CM: High dose norepinephrine-induced apoptosis in cultured rat cardiac fibroblast. Int J Cardiol 136: 33-39, 2009.

4. Lameris TW, de Zeeuw S, Alberts G, Boomsma F, Duncker DJ, Verdouw PD, Veld AJ and van Den Meiracker AH: Time course and mechanism of myocardial catecholamine release during transient ischemia in vivo. Circulation 101: 2645-2650, 2000.

5. Fu YC, Chi CS, Yin SC, Hwang B, Chiu YT and Hsu SL: Norepinephrine induces apoptosis in neonatal rat cardiomyocytes through a reactive oxygen species-TNF alpha-caspase signaling pathway. Cardiovasc Res 62: 558-567, 2004.

6. Thakur A, Alam MJ, Ajayakumar MR, Ghaskadbi S, Sharma M and Goswami SK: Norepinephrinr-induced apoptotic and hypertrophic responses in $\mathrm{H} 9 \mathrm{c} 2$ cardiac myoblasts are characterized by different repertoire of reactive oxygen species generation. Redox Bio 5: 243-252, 2015.

7. Wan C, Chen Y, Yin P, Han D, Xu X, He S, Liu M, Hou X, Liu F and $\mathrm{Xu}$ J: Transport stress induces apoptosis in rat myocardial tissue via activation of the mitogen-activated protein kinase signaling pathways. Heart Vessels 31: 212-221, 2014.

8. You ZL, Xia Q, Liang FR, Tang YJ, Xu CL, Huang J, Zhao L, Zhang WZ and He JJ: Effects on the expression of GABAA receptor subunits by jujuboside A treatment in rat hippocampal neurons. J Ethnopharmacol 128: 419-423, 2010.

9. Liu Z, Zhao X, Liu B, Liu AJ, Li H, Mao X, Wu B, Bi KS and Jia Y: Jujuboside A, a neuroprotective agent from semen Ziziphi Spinosae ameliorates behavioral disorders of the dementia mouse model induced by A $\beta$ 1-42. Eur J Pharmacol 738: 206-213, 2014

10. Cao JX, Zhang QY, Cui SY, Cui XY, Zhang J, Zhang YH, Bai YJ and Zhao YY: Hypnotic effect of jujubosides from Semen Ziziphi Spinosae. J Ethnopharmacol 130: 163-166, 2010.

11. Wang XX, Ma GI, Xie JB and Pang GC: Influence of JuA in evoking communication changes between the small intestines and brain tissues of rats and the GABAA and GABAB receptor transcription levels of hippocampal neurons. J Ethnopharmacol 159: 215-223, 2015

12. Han D, Wan C, Liu F, Xu X, Jiang L and Xu J: Jujuboside A protects $\mathrm{H} 9 \mathrm{C} 2$ cells from isoproterenol-induced injury via activating PI3K/Akt/mTOR signaling pathway. Evid Based Complement Alternat Med 2016: 9593716, 2016.

13. Livak KJ and Schmittgen TD: Analysis of relative gene expression data using real-time quantitative PCR and the 2(-Delta Delta C(T)) method. Methods 25: 402-408, 2001.

14. Fu YC, Chi CS, Yin SC, Hwang B, Chiu YT and Hsu SL: Norepinephrine induces apoptosis in neonatal rat endothelial cells via down-regulation of Bcl-2 and activation of beta-adrenergic and caspase-2 pathways. Cardiovasc Res 61: 143-151, 2014.

15. Kohli S, Chhabra A, Jaiswal A, Rustagi Y, Sharma M and Rani V: Curcumin suppresses gelatinase B mediated norepinephrine induced stress in H9c2 cardiomyocytes. PLoS One 8: e76519, 2013.

16. Zhang C, Shan XL, Liao YL, Zhao P, Guo W, Wei HC and Lu R: Effects of stachydrine on norepinephrine-induced neonatal rat cardiac myocytes hypertrophy and intracellular calcium transients. BMC Complement Altern Med 14: 474, 2014.

17. Jain A, Atale N, Kohli S, Bhattacharya S, Sharma M and Rani V: An assessment of norepinephrine mediated hypertrophy to apoptosis transition in cardiac cells: A signal for cell death. Chem Biol Interact 225: 54-62, 2015 .

18. Gao QH, Wu CS and Wang M: The jujube (Ziziphus jujuba Mill.) fruit: A review of current knowledge of fruit composition and health benefits. J Agr Food Chem 61: 3351-3363, 2013.

19. Shou CH, Wang J, Zheng XX and Guo DW: Inhibitory effect of jujuboside $\mathrm{A}$ on penicillin sodium induced hyperactivity in rat hippocampal CA1 area in vitro. Acta Pharmacol Sin 22: 986-990, 2001.

20. Communal C, Singh K, Pimentel DR and Colucci WS: Norepinephrine stimulates apoptosis in adult rat ventricular myocytes by activation of the beta-adrenergic pathway. Circulation 98: 1329-1334, 1998.

21. Lajevic MD, Suleiman S, Cohen RL and Chambers DA: Activation of p38 mitogen-activated protein kinase by norepinephrine in T-lineage cells. J Immunol 132: 197-208, 2011.

22. Jürgensmeier JM, Xie Z, Deveraux Q, Ellerby L, Bredesen D and Reed JC: Bax directly induces release of cytochrome $\mathrm{c}$ from isolated mitochondria. P Natl Acad Sci USA 95: 4997-5002, 1998.

23. McDonald TE, Grinman MN, Carthy CM and Walley KR: Endotoxin infusion in rats induces apoptotic and survival pathways in hearts. Am J Physiol Heart Circ Physiol 279: H2053-H2061, 2000. 
24. Bratton SB, Walker G, Srinivasula SM, Sun XM, Butterworth M Alnemri ES and Cohen GM: Recruitment, activation and retention of caspases- 9 and -3 by Apaf- 1 apoptosome and associated XIAP complexes. Embo J 20: 998-1009, 2001.

25. Agata N, Ahmad R, Kawano T, Raina D, Kharbanda S and Kufe D: MUC1 oncoprotein blocks death receptor-mediated apoptosis by inhibiting recruitment of caspase-8. Cancer Res 68 : 6136-6144, 2008.

26. Funakoshi T, Aki T, Nakayama H, Watanuki Y, Imori S and Uemura K: Reactive oxygen species-independent rapid initiation of mitochondrial apoptotic pathway by chelerythrine. Toxicol In vitro 25: 1581-1587, 2011.

27. Liu L, Zhang Z and Xing D: Cell death via mitochondrial apoptotic pathway due to activation of Bax by lysosomal photodamage. Free Radical Bio Med 51: 53-68, 2011.

28. Nakagawa T, Zhu H, Morishima N, Li E, Xu J, Yankner BA and Yuan J: Caspase-12 mediates endoplasmic-reticulum-specific apoptosis and cytotoxicity by amyloid-beta. Nature 403: 98-103, 2000.

29. Zhu W, Zou Y, Aikawa R, Harada K, Kudoh S Uozumi H, Hayashi D, Gu Y, Yamazaki T, Nagai R, et al: MAPK superfamily plays an important role in daunomycin-induced apoptosis of cardiac myocytes. Circulation 100 2100-2107, 1999

30. Chang F, Liu J, Fu H, Wang J, Li F, Yue H, Li W, Zhao J and Yin D: GSK-3 $\beta$ promotes PA-induced apoptosis througu changing $\beta$-arrestin 2 nucleus location in H9c2 cardiomyocytes. Apoptosis 21: 1045-1055, 2016.
31. Wang G, Cui J, Guo Y, Wang Y, Kang L and Liu L: Cyclosporin A protectes $\mathrm{H} 9 \mathrm{c} 2$ cells against chemical hypoxia-induced injury via inhibition of MAPK signaling pathway. Int Heart J 57: 483-489, 2016

32. Liu Y, Zhang S, Su D, Liu J, Cheng Y, Zou L, Li W and Jiang Y: Inhibiting (pro)renin receptor-mediated p38 MAPK signaling decreases hypoxia/reoxygenation-induced apoptosis in H9c2 cells. Mol Cell Biochem 403: 267-276, 2015.

33. Song YH, Cai H, Zhao ZM, Chang WJ, Gu N, Cao SP and Wu ML: Icariin attenuated oxidative stress induced-cardiac apoptosis by mitochondria protection and ERK activation. Biomed Pharmacother 83: 1089-1094, 2016.

34. Baines CP and Molkentin JD: STRESS signaling pathways that modulate cardiac myocyte apoptosis. J Mol Cell Cardiol 38: 47-62, 2005.

35. Ma L, Liu H, Xie Z, Yang S, Xu W, Hou J and Yu B: Ginsenoside $\mathrm{Rb} 3$ protects cardiomyocytes against ischemia-reperfusion injury via the inhibition of JNK-mediated NF- $\kappa$ B pathway: A mouse cardiomyocyte model. PLoS One 9: e103628, 2014

36. Downward J: PI3-kinase, Akt and cell survival. Semin Cell Dev Biol 15: 177-182, 2004

37. Yin P, Xu J, He S, Liu F, Yin J, Wan C, Mei C, Yin Y, Xu X and $\mathrm{Xia} Z$ : Endoplasmic reticulum stress in heat- and shake-induced injury in the rat small intestine. PLoS One 10: e0143922, 2015.

38. Wang X, Chen WR and Xing D: A pathway from JNK through decreased ERK and Akt activities for FOXO3a nuclear translocationin response to UV irradiation. J Cell Physiol 227: 1168-1178, 2011. 